\title{
THE FORMAL LINEARIZATION OF A SEMISIMPLE LIE ALGEBRA OF VECTOR FIELDS ABOUT A SINGULAR POINT $\left({ }^{1}\right)$
}

\author{
BY \\ ROBERT HERMANN( $\left.{ }^{2}\right)$
}

\begin{abstract}
A classical theorem by Poincaré gives conditions that a nonlinear ordinary differential equation

$$
d x / d t=A(x)
$$

with $A(0)=0$ in $n$ variables $x=\left(x_{1}, \ldots, x_{n}\right)$ can be reduced to a linear form

$$
\frac{d x^{\prime}}{d t}=\frac{\partial A}{\partial x}(0) x^{\prime}
$$
\end{abstract}

by a change of variables $x^{\prime}=f(x)$. A generalization is given for a finite set of such differential equations, which form a semisimple Lie algebra.

I. Introduction. Palais and Smale have posed the following problem: suppose that $G$ is a connected semisimple Lie group of transformations on a manifold $M$. Let $p$ be a fixed point for $G$. Is it possible to choose a coordinate system in the neighbourhood of $p$ such that the action of $G$ is linear in these coordinates? If $G$ is compact, this is well known, and this problem can be considered as a first step towards a broad programme (suggested also by Palais and Smale) of investigating the global properties of semisimple transformation groups by the methods of the modern theory of dynamical systems and differential topology that have proved successful in studying compact and Abelian transformation groups.

Since $G$ is connected, the question can be reduced to the study of a semisimple Lie algebra of vector fields in the neighbourhood of a zero point for all the vector fields in the algebra. Now, in the case of a single vector field which is real analytic, there is a well-known classical theory of linearization in the neighbourhood of a singular point, starting with Poincaré's work. Crudely, it has two aspects: finding the linearizing diffeomorphism as a formal power series, then proving that it converges. In this paper, we shall present the construction of the formal power series for the semisimple case, where all the relevant cohomology groups are automatically zero. The second and, of course, much more difficult part of the problem, i.e., showing the actual existence of a linearizing change of coordinates,

Received by the editors November 29, 1966.

(1) This work was supported by the National Science Foundation.

${ }^{(2)}$ University of California, Santa Cruz, and Stanford Linear Accelerator Center. 
will not be considered in this paper, although we might remark that the methods developed by Sternberg [2] for the classical case seem to be relevant.

I would like to thank Professor J. Prentki and the Theoretical Study Division at CERN, for hospitality while this paper was written.

II. Lie series and one-parameter groups of diffeomorphisms. Let $M$ be a manifold, with $V(M)$ its Lie algebra of $\left(C^{\infty}\right)$ vector fields and $F(M)$ its ring of $\left(C^{\infty}\right)$ real-valued functions. Each $X \in V(M)$ is then a derivation $f \rightarrow X(f)$ of $F(M)$. Let $t \rightarrow \phi_{t}$ be a one-parameter group of diffeomorphisms of $M$. Then the vector field $W$ defined as follows is the infinitesimal generator of the group

1

$$
W(f)=\left.(\partial / \partial t) \phi_{t}^{*}(f)\right|_{t=0}
$$

for $f \in F(M)$.

Let $X$ be another vector field on $M$, and let $X_{t}$ be the vector field obtained by transforming $X$ under the diffeomorphism $\phi_{t}$. Then

$$
X_{t}(f)=\phi_{t}^{*} X \phi_{t}^{-1 *}(f)
$$

for $f \in F(M)$.

The Taylor series of $t \rightarrow X_{t}$ as a function of $t$ is then

$$
X+t[W, X]+\left(t^{2} / 2 !\right)[W,[W, X]]+\cdots=\exp (\operatorname{Ad} t W)(X) .
$$

This is a Lie series. Its formal algebraic nature enables us to define exp (Ad $t W)(X)$ as a formal series if $W$ is a formal series

$$
W_{1}+W_{2}+\cdots
$$

Notice also that, inherent in the formalism, the correspondence

$$
X \rightarrow \exp (\operatorname{Ad} W)(X)
$$

preserves Jacobi brackets even if the series do not converge.

Finally, note that, even if $W$ is a formal power series, $\exp (\operatorname{Ad} W)(X)$ can be considered as obtained from $X$ in local coordinates by a formal power series type of change of coordinates, such as considered by Poincaré and Birkhoff in their classical work on normal forms of differential equations.

III. Normal forms of Lie algebras of vector fields under formal power series change of coordinates. Suppose $\boldsymbol{G}$ is a Lie algebra of vector fields on $M$ that vanish at the point $p$. We say that $\boldsymbol{G}$ can be formally linearized in a neighbourhood of $p$ if there exists a formal series $W=W_{1}+W_{2}+\cdots$ of vector fields such that:

(a) $W_{1}, W_{2}, \ldots$ vanish at $p$;

(b) there is a coordinate system in a neighbourhood of $p$ such that, for each $X \in G, \exp (\operatorname{Ad} W)(X)$ is a linear vector field in these coordinates.

Let us proceed to the algorithm for constructing $W$. Let $\left(x_{i}\right), i=1, \ldots, n$ be any coordinate system valid in a neighbourhood of $p$, such that $x_{i}(p)=0, i=1, \ldots, n$. 
For each integer $j$, let $V^{j}$ be the set of vector fields whose coefficients are polynomials of degree $j$ in these coordinates. Then,

$$
\left[V^{j}, V^{k}\right] \subset V^{j+k-1} .
$$

For $X \in \boldsymbol{G}$, let

$$
\sum_{j=1}^{\infty} X^{j}, \quad X^{j} \in V^{j}
$$

be the Taylor expansion of the coefficients of $X$ in these coordinates. Then

$$
\left[X^{1}, Y^{1}\right]=[X, Y]^{1}
$$

for $X, Y \in \boldsymbol{G}$, i.e., the $\left\{X^{1}: X \in \boldsymbol{G}\right\}=\boldsymbol{G}^{1}$ forms a Lie algebra of linear vector fields which is a homomorphic image of $G$. (This is just the linear isotropy algebra, of course.) Also,

$$
\left[G^{1}, V^{j}\right] \subset V^{j}
$$

for $j=2,3, \ldots$ i.e., the Jacobi bracket defines a linear representation of $\boldsymbol{G}$ in the finte-dimensional vector space $V^{j}, j=1,2, \ldots$, which we shall denote by $\phi_{j}$. Put:

$$
\omega_{j}(X)=X^{j}
$$

for each $X \in \boldsymbol{G}$.

Then, each $\omega_{j}$ is a 1-cochain of $\boldsymbol{G}$, with coefficients in the representation $\phi_{j}$ of $\boldsymbol{G}$ (see [1] for the details about Lie algebra cohomology).

Now we have two possible ways of defining $W$ : we can look for it in the form $W=W_{2}+W_{3}+\cdots, W_{j} \in V^{j}$, or we can look for $W_{j}^{\prime} \in V^{j}, j=2,3, \ldots$, such that:

(III.2) $\exp (\operatorname{Ad} W)$ is written as a formal product $\ldots \exp \operatorname{Ad} W_{3} \cdot \exp \operatorname{Ad} W_{2}$.

[Note that the Campbell-Baker-Hausdorff formula and the commutation relations (III.1) show that any infinite product of the form (III.2) can be written as exp Ad $W$, for a well-defined formal power series $W$. The point is that the Lie algebra $V^{2}+V^{3}+\cdots$ is a sort of infinite-dimensional version of a nilpotent Lie algebra, in the sense that commutators of a large number of elements have a large degree of homogeneity.] We shall use the second method because it is algebraically simpler. Presumably the first method is preferable for an actual convergence proof, especially since it is closer to the one used by Poincaré.

Now, for $X, Y \in G$,

$$
\begin{aligned}
{[X, Y] } & =\left[X^{1}+X^{2}+\cdots, Y^{1}+Y^{2}+\cdots\right] \\
& =\left[X^{1}, Y^{1}\right]+\left[X^{1}, Y^{2}\right]+\left[X^{2}, Y^{1}\right]+\cdots \\
& =[X, Y]^{1}+[X, Y]^{2}+\cdots
\end{aligned}
$$

i.e.,

$$
\begin{aligned}
\omega_{2}([X, Y]) & =\left[X^{1}, Y^{2}\right]+\left[X^{2}, Y^{1}\right] \\
& =\phi_{2}(X)\left(\omega_{2}(Y)\right)-\phi_{2}(Y)\left(\omega_{2}(X)\right) .
\end{aligned}
$$


This expresses the fact that $d \omega_{2}=0$, i.e., $\omega_{2}$ is a 2-cocycle. If $H^{1}\left(G, \phi_{2}\right)=0$, then there exists $W^{2} \in V^{2}$ such that

$$
\begin{aligned}
\omega_{2}(X) & =d \omega^{2}(X)=\phi_{2}(X)\left(W^{2}\right) \\
& =\left[X^{1}, W^{2}\right] \text { for all } X \in \boldsymbol{G} .
\end{aligned}
$$

Thus,

$$
\begin{aligned}
\exp \left(\operatorname{Ad} W^{2}\right)(X) & =X+\left[W^{2}, X\right]+\cdots \\
& =X^{1}+X^{2}+\cdots+\left[W^{2}, X^{1}\right]+\left[W^{2}, X^{2}\right]+\cdots \\
& =X^{1}\left[X^{2}, W^{2}\right]+\left[W^{2}, X^{2}\right]+\cdots \\
& =X^{1}+\left(\text { terms in } V^{3}+V^{4}+\cdots\right)
\end{aligned}
$$

Now the argument can be repeated with $\exp \left(\operatorname{Ad} W^{2}\right)(\boldsymbol{G})$ replacing $\boldsymbol{G}$. Here $\omega_{2}$ is zero, hence $\omega_{3}$ is a cocycle. Hence, if $H^{1}\left(G, \phi_{3}\right)=0, W^{3}$ can be defined in a similar way. Continuing, we see that $W^{1}, W^{3}, \ldots$ can all be defined in this way, and $W$ can be chosen by (III.2). Of course, if $\boldsymbol{G}$ is semisimple, it is a general theorem that all these cohomology groups vanish. Summing up, we have proved

THEOREM III.1. Suppose $\boldsymbol{G}$ is a semisimple Lie algebra of vector fields on a manifold which have a common zero point. Then, all the vector fields in $G$ can be simultaneously linearized by a formal power series change of coordinates.

IV. Linearizations determined by linearization of a subalgebra. Palais and Smale have also posed the following question: suppose $\boldsymbol{G}$ is a semisimple Lie algebra of vector fields with a fixed point $p$. Suppose a coordinate system is given in which a subalgebra $\boldsymbol{H}$ of $\boldsymbol{G}$ is already linear. What property must the subalgebra have to guarantee that the rest of the vector fields in $\boldsymbol{G}$ are also linear in this same coordinate system?

Keeping the notation of the last section, $\boldsymbol{H}$ already linear means that $\omega_{j}(\boldsymbol{H})=0$, $j=2,3, \ldots$. Start off with $j=2$. Suppose $\omega_{2}=d W^{2}$, for $W^{2} \in V^{2}$. Then

$$
\phi=\omega_{2}(X)=\left[X^{1}, W^{2}\right]
$$

for $X \in \boldsymbol{H}$. Suppose that $\phi_{2}(\boldsymbol{H})$ has no invariant vectors in $V^{2}$. Then, $W^{2}=0$, hence also $\omega_{2}=0$. The argument can be iterated with $\omega_{3}$, which is now a cocycle, since $\omega_{2}$ vanishes, etc. We have then proved

THEOREM IV.1. If $\phi_{j}(\boldsymbol{H})$ has no invariant vectors, and if $\boldsymbol{G}$ is semisimple (or if all the relevant cohomology groups vanish) then, $\boldsymbol{G}$ is linear in the coordinates for which $\boldsymbol{H}$ is linear.

For example, suppose $\boldsymbol{H}^{1}$ contains a vector field of the form

$$
X^{1}=\sum_{i} x_{i} \frac{\partial}{\partial x_{i}}
$$

Suppose $Y=\sum f_{i}(x)\left(\partial / \partial x_{j}\right)$, where each $f_{i}$ is homogeneous of degree $j$, i.e., $Y \in V^{j}$. 
Then

$$
\begin{aligned}
{[X, Y] } & =\sum X\left(f_{i}\right) \frac{\partial}{\partial x_{i}}-f_{i} \frac{\partial}{\partial x_{i}} \\
& =\sum(j-1) f_{i} \frac{\partial}{\partial x_{i}}
\end{aligned}
$$

i.e., if $j \geqq 2, \phi_{j}(\boldsymbol{H})$ has no invariant vectors.

As a final remark, we may notice that the classical linearization theorem of Poincare for a single vector field also falls into this pattern: if $\boldsymbol{G}$ is spanned by a single vector field $X$, if

$$
X=\sum_{i} g_{i} \frac{\partial}{\partial x_{i}}
$$

then

$$
X^{1}=\sum_{i, k} \frac{\partial g_{i}}{\partial x_{k}}(0) x_{k} \frac{\partial}{\partial x_{i}}
$$

Poincaré's condition is that the matrix

$$
\left(\partial g_{i} / \partial x_{k}\right)(0)
$$

has eigenvalues with all positive real parts. However, this condition implies that $H^{1}\left(G, \phi_{j}\right)=0, j=2,3, \ldots$

\section{REFERENCES}

1. N. Jacobson, Lie algebras, Interscience, New York, 1962.

2. S. Sternberg, Local contractions and a theorem of Poincaré, Amer. J. Math. 79 (1959), 809-824.

UNIVERSity OF CALIFORNIA,

Santa Cruz, California 